\title{
Visualizing the Effects of Parameter Variability on Comparative LCA Results
}

\author{
Céline Alexandre, Elisabeth van Overbeke, Maxime Dupriez, \\ Johan Lhotellier and Bernard De Caevel
}

\begin{abstract}
Bar charts and other usual ways of presenting LCA results depict one average or typical situation, lacking to represent the diversity of individual cases and the uncertainties associated to input data or modelling assumptions. This paper presents ways of visualizing variable results in comparative LCA. The main concept is to perform at once calculations representing this variability. Based on Monte Carlo analysis, the approach is enabled by the LCA software RangeLCA, developed by RDC Environment. Results of all simulations can be plotted in function of one or two influential parameters. A clear and complete view can hence be obtained as well as more reliable conclusions. Instead of answering the question "Is a system better than another one in specific cases?", the presented graphs help LCA studies to answer a much broader question: "In which range of situations is a system better than another?".
\end{abstract}

\section{Introduction}

In most LCAs, some parameters are better characterized by variable values than by fixed "typical" values. The reasons for the variability can be of two types: uncertainty and diversity of situations. The uncertainty can be either systematic (e.g. linked to imprecision of the measuring instrument), stochastic (e.g. fluctuation of a parameter with time) or epistemic (e.g. modelling required in the absence of measurement) [1]. The diversity of potential situations within the studied system also leads to parameter variability, e.g. the transport to various customers is best modelled by a range of distances or a waste can be either incinerated or landfilled.

The way the input data variability influences the results can be analysed. The uncertainty analysis is the "systematic procedure to quantify the uncertainty introduced in the results of a life cycle inventory analysis due to the cumulative effects of model imprecision, input uncertainty and data variability" [2]. It is

C. Alexandre · E. van Overbeke $(\varangle) \cdot$ M. Dupriez $\cdot$ J. Lhotellier · B. De Caevel

RDC Environment, 1160 Brussels, Belgium

e-mail: elisabeth.vanoverbeke@ rdcenvironment.be

(C) The Author(s) 2018

E. Benetto et al. (eds.), Designing Sustainable Technologies,

Products and Policies, https://doi.org/10.1007/978-3-319-66981-6_38 
currently mostly performed by Monte Carlo analysis, which is available in several commercial LCA software tools [1].

Sensitivity analysis is defined as a "systematic procedure for estimating the effects of the choices made regarding methods and data on the outcome of a study" [2]. It helps identifying the most influential parameters, for prioritizing data refining and reinforcing conclusions. Sensitivity analyses are in current practice mainly carried out by varying one parameter at a time or by studying alternative scenarios [1].

Wei et al. [3] propose some guidelines on how to perform sensitivity analysis when some data and modelling parts are correlated. Groen et al. [4] discuss several approaches to conduct a sensitivity analysis. They analyse their capacities and drawbacks. They determined that the disadvantage of the method of elementary effects (MEE), using ranges of values from upper to lower boundary of an input parameter, is that "the results are not an estimation of the actual variance decomposition" but MEE can be used as a precursor to the more computationally demanding sampling methods as regression. They see as a main drawback of the standardized regression coefficients (using Monte Carlo sampling) that many runs are needed to calculate the variance decomposition.

In fact, the advantages of using ranges of values for input parameter and Monte Carlo sampling can be combined and the analysis is made easier to interpret by using some fast calculation methods and visual interpretation.

This paper describes the use of Monte Carlo analysis for depicting a large range of possible situations. Emphasis is put on presenting innovative ways of visualizing result variability, which helps interpretation and decision making. The discussion is based on a real case study.

\section{Description of the Case Study}

The selected case study aims at comparing two systems of packaging: a corrugated box versus a reusable folding plastic crate. The functional unit is defined as: the packaging, transport and delivery of goods in one case from the good manufacturer to the distributor or repacker. It is assumed that the cases have similar dimensions in both systems and contain the same number of goods.

For the purpose of this paper, results are only presented for the impact category global warming potential (GWP, with biogenic carbon taken into account). The study is divided into two parts: (i) the "average comparison" scenario, i.e. investigation of the impacts of two average cases, each representative of one system; (ii) the sensitive analysis, i.e. calculation and interpretation of results covering a large range of situations defined as possible for each system. 


\section{RangeLCA Software}

RangeLCA is the own LCA software developed by RDC Environment since the early 90s. It is used here to perform the sensitivity analysis and to propose innovative ways of presenting the variable results.

For every parameter, a probability distribution can be defined to express the parameter variability (either originating in uncertainty or in diversity of situations). Normal, lognormal, uniform, triangular and discrete distributions are available in the software, as well as any mathematically defined distribution. In the case study, a uniform distribution of probability is modelled within the ranges defined by the minimum and maximum values (cf. Table 1). In this example, variability is considered only for modelling parameters but it could also be applied to elementary flows.

RangeLCA performs at once a large number of simulations (3000 in the case study). For each simulation, it selects a random value for each variable parameter, based on its defined probability distribution. This Monte Carlo analysis allows all existing cases to be statistically modelled in a single LCA model. When common to several systems, a parameter takes at each simulation the same value for all the systems (as what is done in other LCA software tools). With RangeLCA, the parameters can be classified by decreasing order of sensitivity of the results to the parameters. Furthermore, the software allows the user to access the results and the parameter values for each Monte Carlo simulation. The variable results can then be plotted against selected parameters.

\section{Presentation of the Results}

\subsection{Average Results}

Results obtained for the defined average cases are presented in Fig. 1 for both compared systems.

Table 1 Case study - values of the main variable parameters

\begin{tabular}{l|l|l|l|l|r}
\hline Parameter & System & Unit & $\begin{array}{l}\text { Average } \\
\text { case }\end{array}$ & Min & Max \\
\hline Height of box & Both & $\mathrm{cm}$ & 20 & 3.5 & 35 \\
\hline Number of uses per crate & Crate & $\#$ & 71 & 1 & 100 \\
\hline Washing rate & Crate & $\%$ & 8.3 & 0 & 100 \\
\hline $\begin{array}{l}\text { Recovery transport (Sending back the crates to a } \\
\text { new good producer) }\end{array}$ & Crate & $\mathrm{km}$ & 200 & 0 & 1000 \\
\hline $\begin{array}{l}\text { Collection transport (Sending the dirty/damaged } \\
\text { crates to service centre) }\end{array}$ & Crate & $\mathrm{km}$ & 200 & 0 & 1000 \\
$\begin{array}{l}\text { Relocation transport (Dispatching the washed } \\
\text { crates) }\end{array}$ & Crate & $\mathrm{km}$ & 200 & 0 & 1000 \\
\hline
\end{tabular}

The total logistic transport for crates combines recovery, collection and relocation transports 


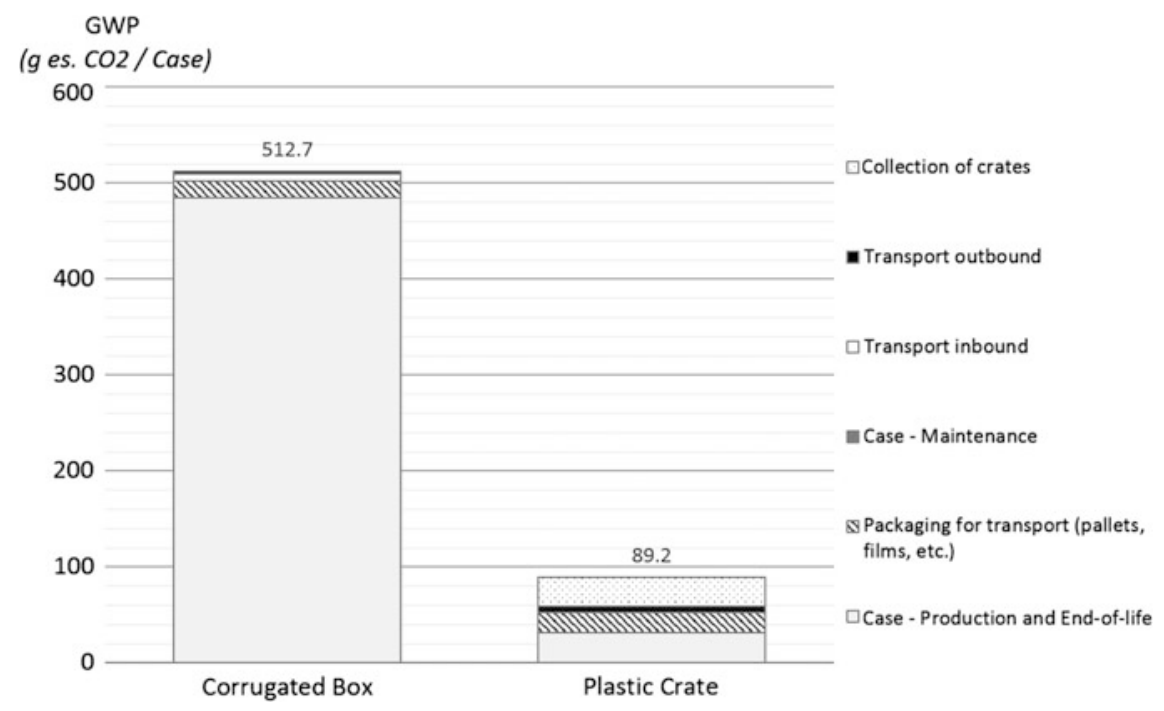

Fig. 1 GWP results for the comparison of average cases - detail per life cycle steps

This graph allows concluding on the lower impacts of the plastic crate versus the corrugated box for the average scenario. It also highlights the most contributing phases. However, such result presentation does not reflect the uncertainty nor provides indication on how would the results evolve in case of a modification of a main parameter value.

\subsection{Results with Variability}

\subsubsection{Range and Delta Graphs}

In Fig. 2, the GWP results of the 3000 simulations are plotted for the compared systems against the total logistic distance, i.e. the most influencing parameter for the crate system. In this "Range graph", a cloud of points is represented for each system. The slope of the trendline indicates how much the results depend on the parameter taken as x-as. The height of the cloud of points reflects the remaining variability, i.e. the variability that is not explained by this main parameter.

For comparing two systems, the relative positions of both clouds of points is analysed. In Fig. 2, the clouds overlap partially. Hence, it is difficult to determine in which situations the corrugated boxes have higher impacts than the crates.

In order to eliminate the common variability of the compared systems, the results of each simulation can be compared two by two. The differences between both systems calculated for all simulations can be plotted in a "Delta graph", represented in Fig. 3. The analysis of delta results indicates that corrugated boxes are better in 


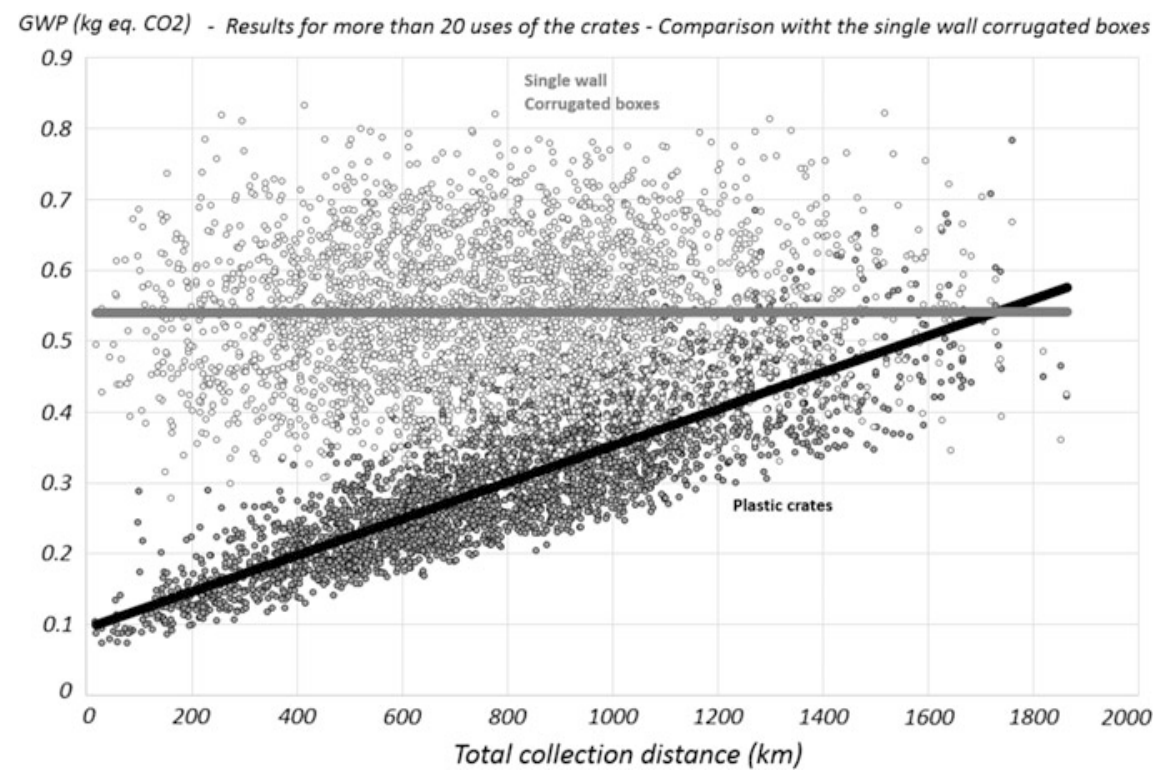

Fig. 2 GWP-variation of results for corrugated boxes and crates in function of the total collection distance

$3 \%$ of the cases studied, as can also be calculated by other software tools. However, the delta graph provides much richer information. It allows drawing specific conclusions for defined ranges of parameter values. In Fig. 3, it appears that up to a distance of $900 \mathrm{~km}$, the corrugated boxes always have higher GWP impacts than plastic crates ("always" means that the conclusion is drawn while keeping all parameters variable or uncertain).

\subsubsection{Areas of Relevance}

The residual variability, beside the one related to the most influential parameter, can be further analysed by looking at a second parameter, for example here the height of the cases. Small incremental ranges of values are defined for both variable parameters (for example, ranges of $1 \mathrm{~cm}$ of height). The results obtained for each combination of height and distance ranges are averaged and compared for both systems. The obtained impact ratios (or indexes) can be represented for all situations, as in Fig. 4, with the level of grey being related to the index (number inside the cells). Conclusions can be drawn within areas of relevance. With an index lower than 1, the light grey cells signify that the plastic crates have a lower impact (the crate is the best solution). The darker the cell, the higher the index. With index above 1, the corrugated boxes have a lower impact (the box is the best solution). A buffer zone is represented in intermediate 


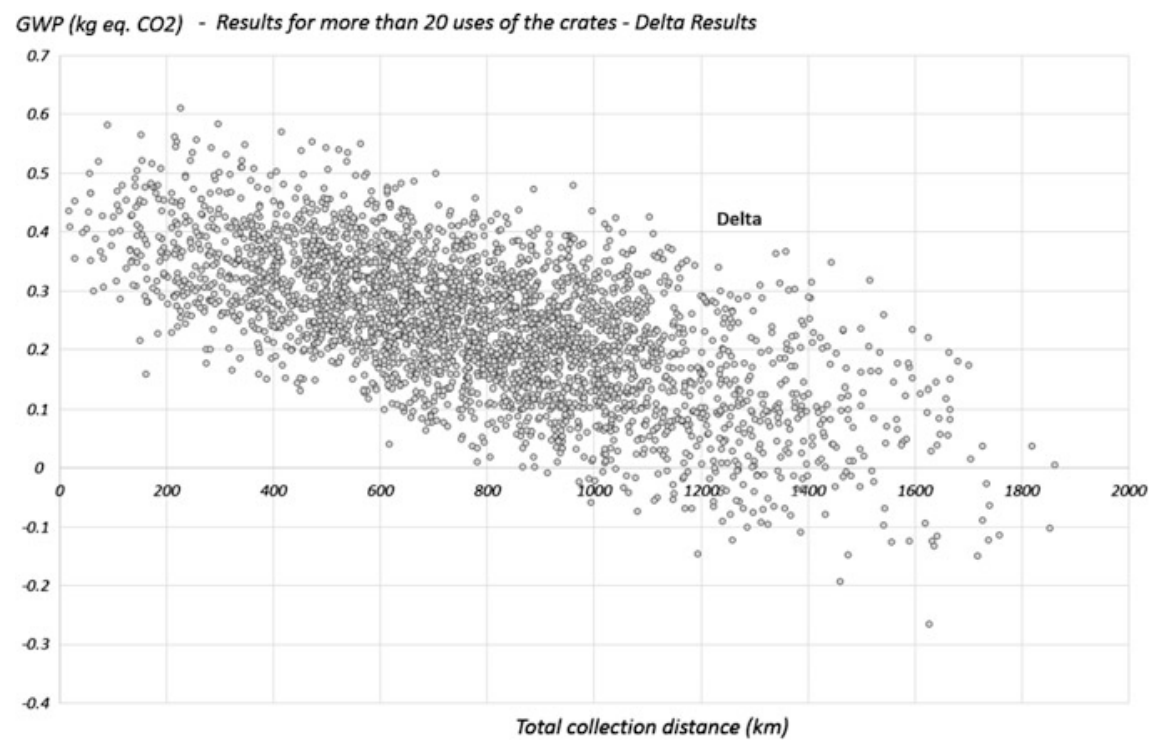

Fig. 3 GWP-variation of the differences between results for corrugated boxes and crates in function of the total collection distance

grey level when the index is around 1. For those cells, it is no clear whether it is the crate or the box that provides the best solution.

\subsection{Discussion}

The GWP results presented for the average cases reveal only that, in the defined specific scenario, crates provide a better solution than boxes, regarding this indicator. Instead, considering the variability of parameters like logistic distance and case height allows identification of the range of situations where this conclusion applies. 


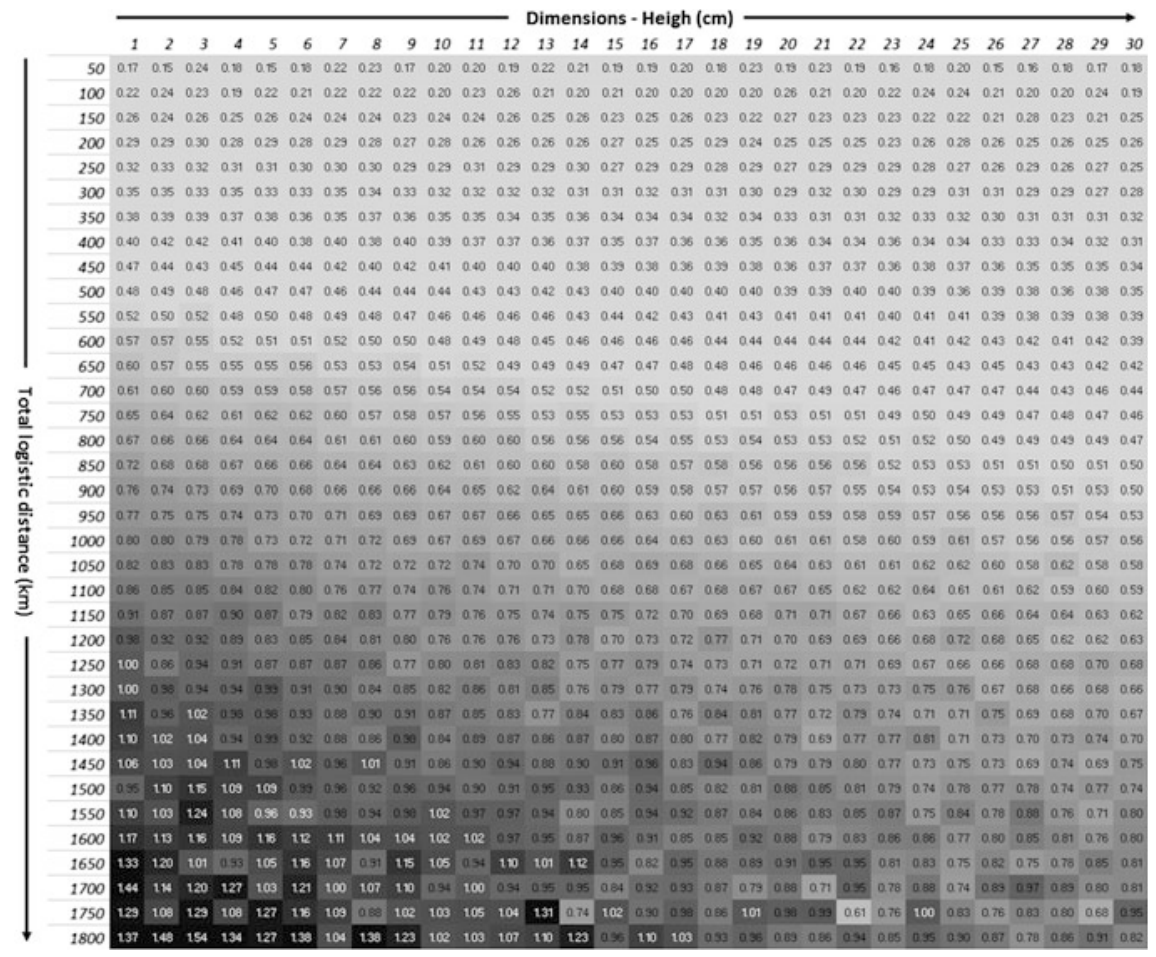

Fig. 4 Ratio of GWP impacts between crates and boxes considering a combined variation of the total logistic distance and the dimension of the cases. An index is visible for each simulation; it is calculated as the ratio between the crate impact and the box impact

\section{Conclusions}

When performing Monte Carlo analysis, it is very helpful to have access to the results of each simulation as well as to the corresponding values of the variable parameters. Thanks to these datasets, made available here by the software RangeLCA, valuable ways of presenting the results have been proposed in the paper. They have been illustrated for one impact category, with the help of a real case study.

Range graphs represent the variable results of each system studied as clouds of points. The sensitivity of the results to a parameter can be highlighted and the amplitude of the variability can be visualized through the cloud shape. This approach plays an important role in iterative LCA in defining priorities for data and model refining. In case of comparative LCA, result differences between two systems can be plotted for all simulations, either against one parameter (delta graphs) and by combining the variability of two parameters (areas of relevance). 
These types of graphs allow acquiring a more complete vision of a complex system. They enhance reliability of conclusions in comparative LCA by showing whether differences are significant or not in terms of sign and amplitude. Instead of answering the question "Is a system better than another one in defined specific cases?", delta graphs and areas of relevance help LCA studies to answer a much broader question: "In which range of situations is a system better than another one?". This is of great help to decision makers.

\section{References}

1. ScoreLCA 2014-03, Igos E, Benetto E, Les différentes sources d'incertitudes en ACV, leurs modes de calcul et impacts sur l'interprétation. June 2015.

2. ISO 14044:2006.

3. Wei W, Larrey-Lassalle P, Faure F, Dumoulin N, Roux P, Mathias J.D, How to conduct a proper sensitivity analysis in life cycle assessment: taking into account correlations within LCI data and interactions within the LCA calculation model, Environ Sci Technol., Vol. 49(1), 2015, pp. 377-85.

4. Groen E.A, Heijungs R, Bokkers E.A.M, de Boer I.J.M, Sensitivity analysis in life cycle assessment, Proceedings of the 9th International Conference on Life Cycle Assessment in the Agri-Food Sector, San Francisco, 2014, http://lcafood2014.org/papers/196.pdf (Accessed 12.06.2017).

Open Access This chapter is licensed under the terms of the Creative Commons Attribution 4.0 International License (http://creativecommons.org/licenses/by/4.0/), which permits use, sharing, adaptation, distribution and reproduction in any medium or format, as long as you give appropriate credit to the original author(s) and the source, provide a link to the Creative Commons license and indicate if changes were made.

The images or other third party material in this chapter are included in the chapter's Creative Commons license, unless indicated otherwise in a credit line to the material. If material is not included in the chapter's Creative Commons license and your intended use is not permitted by statutory regulation or exceeds the permitted use, you will need to obtain permission directly from the copyright holder.

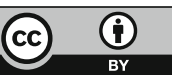

